\title{
HARDWARE IMPLEMENTATION OF THE DISCRETE FOURIER TRANSFORM WITH NON-POWER-OF-TWO PROBLEM SIZE
}

\author{
Peter A. Milder, Franz Franchetti, James C. Hoe, and Markus Püschel \\ Carnegie Mellon University \\ Department of Electrical and Computer Engineering \\ Pittsburgh, PA, United States
}

\begin{abstract}
In this paper, we examine several algorithms suitable for the hardware implementation of the discrete Fourier transform (DFT) with non-power-of two problem size. We incorporate these algorithms into Spiral, a tool capable of performing automatic hardware implementation of transforms such as the DFT. We discuss how each algorithm can be applied to generate different types of hardware structures, and we demonstrate that our tool is able to produce hardware implementations of non-power-of-two sized DFTs over a wide range of cost/performance tradeoff points.
\end{abstract}

Index Terms - Discrete Fourier transform, high-level synthesis, Field programmable gate arrays, algorithms

\section{INTRODUCTION}

Transforms such as the discrete Fourier transform (DFT) are very commonly used in signal processing, communications, and scientific computing applications. However, they are difficult and time-consuming to implement in hardware because of the large number of algorithmic and datapath options that must be considered in order to tailor the implementation to a particular application's cost and performance requirements. The right choices are highly dependent on the context. These problems are worsened when the transform size is not a power of two; algorithms become less regular and the relationships between algorithm and hardware implementation become more complicated. For these reasons, most existing work on hardware implementations of the DFT with non-power-oftwo problem size focuses on building an implementation to meet a specific application's cost and performance goals, not on creating a space of designs with different cost/performance characteristics.

Contribution. In this paper we utilize a hardware generation framework based on Spiral $[1,2]$ to automatically produce hardware implementations of the DFT with non-powerof-two problem size. We consider several algorithmic options including the Bluestein [3] and mixed radix [4] algorithms, and we examine their applicability to the mathematical framework we use in hardware generation. Because designs are implemented automatically, it is possible to evaluate many more options than would likely be evaluated by hand, and we demonstrate that the combination of options considered allows the generation of a range of designs, each with a different tradeoff between cost and performance metrics.

Related work. Most previous work on hardware implementations of non-power-of-two sized DFTs has focused on producing a solution for a specific situation (a given problem size and performance requirement). For example, [5] and [6] consider the specific problem sizes and performance requirements of the Digital Radio Mondiale (DRM) radio broadcasting standard, while [7] presents an FPGA-based pipeline design of a 3,780 point inverse DFT used in a digital television standard.

\section{BACKGROUND}

In this section, we present relevant background on the discrete Fourier transform and fast Fourier transform algorithms.

Discrete Fourier transform. Computing the discrete Fourier transform on $n$ points is defined as the matrix-vector product $y=\operatorname{DFT}_{n} x$, where $x$ and $y$ are $n$ point input and output vectors (respectively), and

$$
\operatorname{DFT}_{n}=\left[\omega_{n}^{k \ell}\right]_{0 \leq k, \ell<n}, \quad \omega_{n}=e^{-2 \pi i / n} .
$$

Fast Fourier transform. Fast Fourier transform (FFT) algorithms allow the computation of $\mathrm{DFT}_{n}$ in $O(n \log n)$ operations. Using the Kronecker product formalism developed in [4], an FFT algorithm can be written as a formula that represents a factorization of the dense $\mathrm{DFT}_{n}$ matrix into a product of structured sparse matrices. For example, the wellknown Cooley-Tukey FFT can be written as

$$
\mathrm{DFT}_{m n}=\left(\mathrm{DFT}_{m} \otimes I_{n}\right) T_{n}^{m n}\left(I_{m} \otimes \mathrm{DFT}_{n}\right) L_{m}^{m n} .
$$

$L_{m}^{m n}$ represents a stride permutation matrix that permutes its input according to:

$$
\text { in }+j \mapsto j m+i, \quad 0 \leq i<m, 0 \leq j<n .
$$

$I_{m}$ is the $m \times m$ identity matrix, and $\otimes$ is the tensor product, defined:

$$
A \otimes B=\left[a_{k, \ell} B\right], \text { where } A=\left[a_{k, \ell}\right] \text {. }
$$




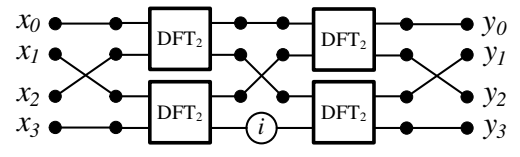

Fig. 1. Dataflow illustration of (1).

Lastly, $T_{n}^{m n}$ is a diagonal matrix of "twiddle factors," (as specified in [8]). Figure 1 shows a dataflow interpretation of the formula (1) when $n=2$ and $m=2$.

Automatic Hardware Generation. Spiral $[1,2]$ is an automated tool for the generation and optimization of hardware and software implementations of transforms such as the DFT. Spiral takes a given transform matrix $\left(\mathrm{DFT}_{n}\right)$ and expands it using one or more FFT algorithms, resulting in a formula. In [1], we extend the Kronecker formalism to enable the description of a rich space of hardware architectures by explicitly specifying sequential reuse of computational elements within the formula. This allows Spiral to automatically generate many possible hardware implementations of a transform of a given size, each with different trade-offs between various costs (e.g., circuit area) and performance metrics (e.g., throughput, latency).

Figure 2(a) shows a datapath with no sequential reuse. It contains three repeated stages, each with four parallel computation blocks labeled $A_{2}$. It processes eight data elements in parallel. Next, Figure 2(b) employs streaming reuse to stream the data vector through the system over multiple cycles at a fixed rate. We call this rate the streaming width (two in this example). ${ }^{1}$ In Figure 2(c), the three computation stages have been collapsed to a single stage using a technique called iterative reuse. Now, the data vector must feed back and pass through the computation block multiple times.

By using different amounts of sequential reuse, different cost/performance trade-offs can be obtained. The more reuse employed, the smaller a datapath will be, but it will be commensurately slower.

\section{NON-POWER-OF-TWO FFT ALGORITHMS}

In this section, we discuss four FFT algorithms that we use with our formula-driven generation framework to implement the DFT with non-power-of-two problem size.

Pease FFT and Iterative FFT. The Pease FFT [11] and Iterative FFT are commonly used FFT algorithms that decompose an $r^{t}$ point DFT into DFTs on $r$ points, where $r$ is called the radix. Both algorithms can be derived from (1). The radix- $r$ Pease and Iterative FFTs are (respectively):

$$
\begin{gathered}
\mathrm{DFT}_{r^{t}}=\left(\prod_{\ell=0}^{t-1} L_{r}^{r^{t}}\left(I_{r^{t-1}} \otimes \mathrm{DFT}_{r}\right) D_{\ell}^{r^{t}}\right) R_{r}^{r^{t}} \\
\mathrm{DFT}_{r^{t}}=\left(\prod_{\ell=0}^{t-1}\left(I_{r^{\ell}} \otimes \mathrm{DFT}_{r} \otimes I_{r^{t-\ell-1}}\right) E_{\ell}^{r^{t}}\right) R_{r}^{r^{t}}
\end{gathered}
$$

\footnotetext{
${ }^{1}$ Permutations on streaming data require the use of memory; we implement them as in $[9,10]$.
}

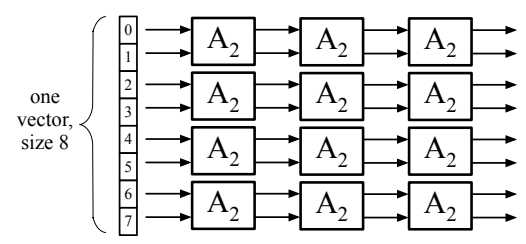

(a) Example combinational datapath.

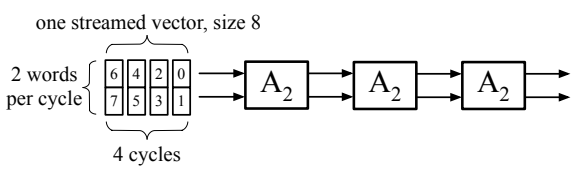

(b) Datapath employing streaming reuse.

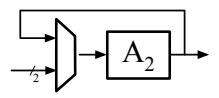

(c) Datapath employing iterative reuse.

Fig. 2. Illustration of sequential reuse techniques. All three perform the same computation, but with differing cost and performance.

Matrix $R_{r}^{r^{t}}$ is the base-r digit reversal permutation on $r^{t}$ points. When $r=2$, this is called the "bit reversal permutation." $D$ and $E$ are diagonal matrices of twiddle factors. The remaining matrices are as defined in Section 2.

Streaming reuse can be applied to both algorithms (as in Figure 2(b)), but only (2) can be used for iterative reuse (as in Figure 2(c)). When using these algorithms to generate streaming hardware, the streaming width must be a power of $r$. So, as $r$ increases, the space of datapaths that can be generated by these formulas decreases.

Mixed radix FFT. The mixed radix FFT algorithm (based on (1)) breaks down a DFT of size $r^{k} s^{\ell}$ into multiple DFTs of sizes $r^{k}$ and $s^{\ell}$ :

$$
\begin{aligned}
& \operatorname{DFT}_{r^{k} s^{\ell}}=L_{r^{k}}^{r^{k} s^{\ell}}\left(I_{s^{\ell}} \otimes \mathrm{DFT}_{r^{k}}\right) L_{s^{\ell}}^{r^{k} s^{\ell}} T_{s^{\ell}}^{r^{k} s^{\ell}} \\
& \cdot\left(I_{r^{k}} \otimes \mathrm{DFT}_{s^{\ell}}\right) L_{r^{k}}^{r^{k} s^{\ell}} .
\end{aligned}
$$

Then, the $\mathrm{DFT}_{r^{k}}$ and $\mathrm{DFT}_{s^{\ell}}$ matrices can be recursively decomposed using radix $r$ and $s$ algorithms (2) or (3). In general, the two stages must be built independently - they can not be collapsed into one iterative reuse stage (as in Figure 2(c)).

The structure of this algorithm imposes an additional restriction on the applicable streaming width: it must evenly divide the problem size $r^{k} \cdot s^{\ell}$, and it must be a multiple of $r \cdot s$. Thus, as the radices $r$ and $s$ get larger, the datapath options become more restricted.

This algorithm can also be applied recursively to break a problem into three or more radices, but as the number of radices increases, the streaming width becomes more restricted, limiting flexibility.

Bluestein FFT. The Bluestein FFT [3] is a convolution-based algorithm for any problem size $n$. The algorithm performs the 
DFT by scaling the input vector and convolving it with precomputed coefficients. The convolution of two $n$ length signals can be performed as point-wise multiplication of length $m>2 n-1$ in the frequency domain. This allows a DFT of any given size to be computed using DFTs of two-power size, at the expense of additional operations. We can view this algorithm as:

$$
\mathrm{DFT}_{n}=D_{n \times m}^{(2)} \mathrm{DFT}_{m}^{-1} D_{m}^{(1)} \mathrm{DFT}_{m} D_{m \times n}^{(0)}
$$

where $m=2^{\left\lceil\log _{2}(n)\right\rceil+1}$ is the smallest power of two greater than $2 n-1$, and the $D$ matrices are diagonal matrices that scale the vector by constant values. $D_{m \times n}^{(0)}$ and $D_{n \times m}^{(2)}$ are rectangular matrices, so in addition to scaling the data, $D^{(0)}$ extends the input data vector from $n$ points to $m$ points (by zero padding), and $D^{(2)}$ shortens the output data vector from $m$ points to $n$ points (by discarding unneeded data).

The Bluestein FFT algorithm has a higher operation count than the other algorithms considered, but its structure is more regular than (4); the forward and inverse $\mathrm{DFT}_{n}$ can be collapsed into one logic block, allowing the datapath to exhibit iterative reuse if desired (as in Figure 2(c)). In Section 4 we will see that this allows the Bluestein algorithm to produce smaller hardware implementations than the mixed radix FFT, while the mixed radix algorithm is able to obtain higher performance at the cost of added logic.

\section{EXPERIMENTAL RESULTS}

In this section, we evaluate the designs generated from (2), (3), (4), and (5). We compare the effects of each algorithm and illustrate how the algorithmic options affect the quality and types of datapaths that can be implemented within our framework. Our experiments evaluate the designs implemented on a Xilinx Virtex-5 FPGA (field-programmable gate array), but the designs produced by our tool are not FPGAspecific; they are also suitable for implementation as an ASIC (application-specific integrated circuit).

Experimental Setup. Given a transform of a specific size, a set of algorithmic options, and hardware directives that specify the desired datapath characteristics, Spiral automatically generates a corresponding pipelined hardware implementation in synthesizable register-transfer level Verilog. In these experiments, we generate designs that operate on 16 bit fixed point data words (for each of the real and imaginary parts of complex data), but Spiral is able to generate designs for any precision fixed-point or single precision floating point.

In these experiments, we generate and evaluate all of the options presented above with a streaming width up to 32 . We use Xilinx ISE to synthesize and place/route each design for a Xilinx Virtex-5 LX 330 FPGA. When a memory structure is needed, we utilize an on-chip block RAM when we will utilize $2 \mathrm{~KB}$ or more, otherwise we build the memory out of FPGA logic elements. (This threshold is also a controllable parameter in our generation tool.)
The set of algorithms and datapaths we consider depends on the problem size. Here, we consider four values of $n$ that illustrate four different classes of problem size. In Figure 3, we examine the throughput performance (in million samples per second) versus FPGA area consumed (in Virtex-5 slices) for each design. A similar tradeoff evaluation can be performed for other cost or performance metrics, such as latency.

Large prime size. First, Figure 3(a) shows throughput versus area for implementations of $\mathrm{DFT}_{499}$. Because 499 is a large prime number, the only applicable option among those we consider is the Bluestein FFT algorithm. Each black circle represents one Bluestein-based datapath, and the black line illustrates the designs in the Pareto-optimal set-the set of best tradeoff points among those evaluated. The slowest design requires approximately 1,500 slices and has a throughput of 11 million samples per second (MSPS). The fastest is $18 \times$ larger but $60 \times$ faster.

Composite number with larger prime factors. Next, Figure 3(b) shows results for $\mathrm{DFT}_{405}$. In addition to the Bluestein algorithm, the mixed radix FFT algorithm applies to this problem; it decomposes $\mathrm{DFT}_{405}$ into $\mathrm{DFT}_{3^{4}}$ and $\mathrm{DFT}_{5}$. This provides two additional designs (with streaming width of $3 \cdot 5=15$ ), shown as white triangles. Although the larger of these designs provides a much higher throughput than a similarly sized Bluestein-derived design, it is important to note that the cross-over point (where the mixed-radix designs become the better choice) is quite large (at approximately 15,000 slices). So, if design requirements require a smaller, lower throughput core, the Bluestein algorithm is a better choice.

Composite number with smaller prime factors. Third, Figure 3(c) illustrates throughput and area for $\mathrm{DFT}_{432}$. Similar to the previous example, $\mathrm{DFT}_{432}$ can be implemented using the mixed radix algorithm. However, unlike $\mathrm{DFT}_{405}$, we can decompose $\mathrm{DFT}_{432}$ into smaller radices: 2 and 3 (because $\left.432=2^{4} \times 3^{3}\right)$. Since the radices are smaller, the mixed radix algorithm can be used with more options for streaming width, leading to a wider set of Pareto-optimal designs than for $\mathrm{DFT}_{432}$. The Bluestein designs are still the best choice for the smallest/slowest designs, but the cross-over point (about 9,000 slices) is lower than in the previous example.

Power of small prime. Lastly, Figure 3(d) shows results for $\mathrm{DFT}_{243}=\mathrm{DFT}_{3}$. Now, the mixed radix algorithm is unneeded; because the problem size is a power of three, the radix-3 Pease and Iterative FFTs are applied directly (shown as white triangles). Here, the cores built with the radix-3 algorithms surpass the performance of the Bluestein cores at a much lower area than in the other problems, since the singleradix design can be built with less logic than in the previous multi-radix designs.

These results show that our framework produces designs with a range of cost/performance trade-offs. However, the range of performance that can be obtained depends on the 
(a) DFT 499 (16 bit fixed point) on Xilinx Virtex-5 FPGA

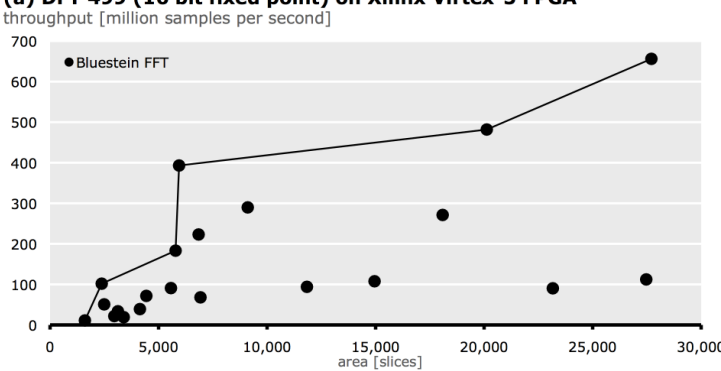

(c) DFT 432 (16 bit fixed point) on Xilinx Virtex-5 FPGA

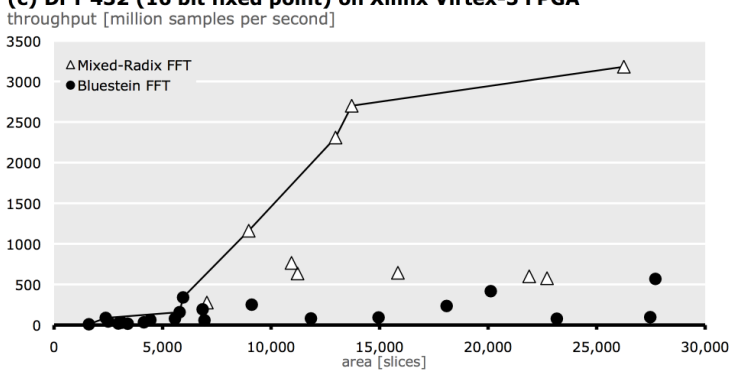

Fig. 3. Throughput (y-axis) versus area (x-axis) for $\mathrm{DFT}_{499}, \mathrm{DFT}_{405}, \mathrm{DFT}_{432}$, and $\mathrm{DFT}_{243}$.

composition of the problem size, which affects the set of algorithms that are applicable.

\section{CONCLUSION}

In this paper, we explore hardware implementation of the discrete Fourier transform with problem sizes that are not powers of two. We discuss several algorithms for this class of problems that fit within our automatic hardware generation framework. We show how different algorithms can be applied to DFTs of different problem sizes, and we discuss the types of hardware structures that may be generated for each. Lastly, we provide an evaluation of designs generated using the algorithmic and datapath options described, and show that the options considered allow the tool to produce designs over a range of cost/performance tradeoff points.

\section{REFERENCES}

[1] P. A. Milder, F. Franchetti, J. C. Hoe, and M. Püschel, "Formal datapath representation and manipulation for implementing DSP transforms," in Proc. Design Automation Conference, 2008, pp. 385-390.

[2] M. Püschel et al., "SPIRAL: Code generation for DSP transforms," Proc. of the IEEE, vol. 93, no. 2, pp. 232275, 2005.

[3] L. I. Bluestein, "A linear filtering approach to computation of discrete Fourier transform," IEEE Tranactions on Audio and Electroacoustics, vol. 18, no. 4, pp. 451-455, 1970.

[4] C. Van Loan, Computational Frameworks for the Fast Fourier Transform, SIAM, 1992.

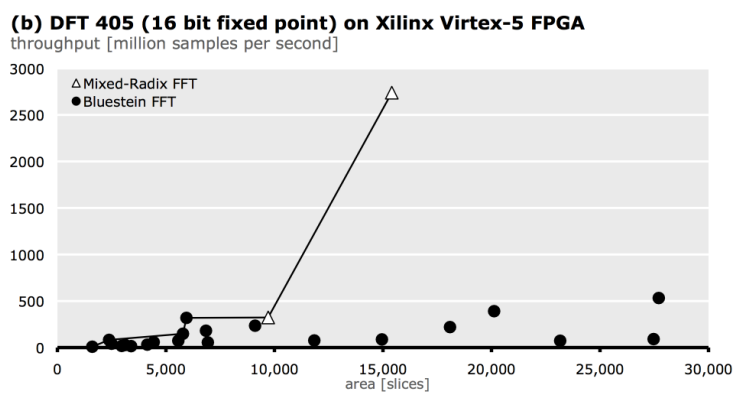

(d) DFT 243 (16 bit fixed point) on Xilinx Virtex-5 FPGA throughput [million samples per second]

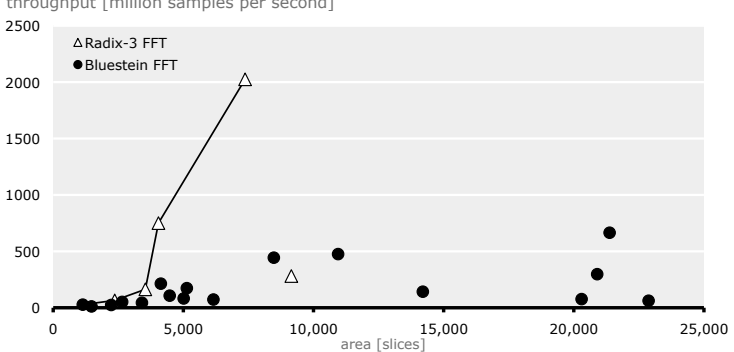

[5] M. D. van de Burgwal, P. T. Wolkotte, and G. J. M. Smit, "Non-power-of-two FFTs: Exploring the flexibility of the Montium TP," International Journal of Reconfigurable Computing, to appear.

[6] D.-S. Kim, S.-S. Lee, J.-Y. Song, K.-Y. Wang, and D.J. Chung, "Design of a mixed prime factor FFT for portable digital radio mondiale receiver," IEEE Transactions on Consumer Electronics, vol. 54, no. 4, pp. 15901594, 2008.

[7] Z.-X. Yang, Y.-P. Hu, C.-Y. Pan, and L. Yang, "Design of a 3780-point IFFT processor for TDS-OFDM," IEEE Transactions on Broadcasting, vol. 48, no. 1, pp. 57-61, 2002.

[8] J. Johnson, R. W. Johnson, D. Rodriguez, and R. Tolimieri, "A methodology for designing, modifying, and implementing Fourier transform algorithms on various architectures," IEEE Trans. Circuits and Systems, vol. 9, pp. 449-500, 1990.

[9] M. Püschel, P. A. Milder, and J. C. Hoe, "Permuting streaming data using RAMs," Journal of the ACM, vol. 56, no. 2, pp. 10:1-10:34, 2009.

[10] P. A. Milder, J. C. Hoe, and M. Püschel, "Automatic generation of streaming datapaths for arbitrary fixed permutations," in Proc. Design, Automation and Test in Europe, 2009, pp. 1118-1123.

[11] M. C. Pease, "An adaptation of the fast Fourier transform for parallel processing," Journal of the ACM, vol. 15, no. 2, April 1968. 